\title{
Evaluación del aprendizaje de los estudiantes en los tres primeros cursos del Grado de Medicina de la Universitat de Barcelona
}

José M. Vidal-Taboada, Jordi Palés-Argullós, Josep Saura

Introducción. El proceso de adaptación al Espacio Europeo de Educación Superior ha comportado cambios en los sistemas de evaluación de los aprendizajes en las facultades de medicina y ha introducido los conceptos de evaluación de competencias y evaluación continuada.

Objetivos. Describir y analizar, después de seis años de implementación del nuevo plan de estudios de medicina, cómo se está realizando el proceso de evaluación continuada en las diferentes asignaturas de los tres primeros cursos del Grado de Medicina de la Universitat de Barcelona, comparándolo con lo recomendado por los expertos en evaluación en educación médica, y establecer posibles mejoras.

Sujetos y métodos. Se describen las actividades evaluativas e instrumentos utilizados por las diferentes asignaturas que demuestran una gran variabilidad y diversificación y una evaluación compartimentada. Se recogen las opiniones y el grado de satisfacción de los coordinadores sobre las actividades evaluativas utilizadas. Asimismo, se evidencia el esfuerzo realizado por los profesores de las distintas asignaturas para promover mejoras en el sistema.

Resultados. Se comparan los resultados obtenidos con las recomendaciones establecidas por los expertos en educación médica, con especial referencia a los programas de formación institucional y al paradigma de la evaluación para el aprendizaje. Se discuten las dificultades existentes para desarrollar un mejor sistema de evaluación continuada.

Conclusiones. A pesar del esfuerzo realizado y de las mejoras introducidas en las actividades de evaluación, estas no se ajustan totalmente a lo que debería ser una evaluación continuada real; se aboga por promover programas de formación del profesorado sobre evaluación en educación médica.

Palabras clave. Evaluación continuada. Evaluación de competencias. Grado de Medicina.

\section{Student's learning assessment in the first three years of the Degree of Medicine in the University of Barcelona}

Introduction. The process of adaptation to the European Higher Education Area has brought changes in the assessment of learning outcomes in medical schools and has introduced the concepts of competence assessment and continuous assessment.

Aims. To describe and analyze, six years after the implementation of the new curriculum, how the continuous assessment process is being carried out in the different subjects of the first three years of the Degree of Medicine at the University of Barcelona, comparing it with what is recommended by the experts in assessment and to propose ways to improve it.

Subjects and methods. The different assessment activities and tools used in the different subjects are described, showing a great variability and diversification and a compartmentalized assessment. The opinions and level of satisfaction of the coordinators of different subjects regarding the assessment activities used are also shown. Likewise, it is evidenced the effort made by the teachers in promoting improvements in the system.

Results. The results obtained are compared with the recommendations established in the literature by the experts in medical education, with special reference to the concept of programmatic and institutional assessment and the paradigm of the assessment for learning. The existing difficulties to develop a better continuous assessment are discussed.

Conclusions. Despite the efforts made and the improvements introduced in the assessment activities, these do not totally conform to what a real continuous assessment must be and we advocate to promote teacher's training programs on assessment in medical education.

Key words. Continuous assessment. Degree of Medicine. Learning outcomes assessment.
Departamento de Biomedicina Facultad de Medicina y Ciencias de la Salud. Universitat de Barcelona. Barcelona, España.

Correspondencia: Dr. Josep Saura. Unidad de Bioquímica i Biología Molecular. Departamento de Biomedicina. Facultad de Medicina. Universitat de Barcelona. Casanova, 143, planta 3. E-08036 Barcelona.

E-mail:

josepsaura@ub.edu

Agradecimientos:

A todos los coordinadores de las asignaturas de los cursos primero, segundo y tercero del Grado de Medicina del Campus Clínic, por su disponibilidad y participación: R.M Aligué, B. Campos, M. Canals, C. Enrich, R. Farré, S. Ginés, J.M. Grau, C. Junqué, A. Lafuente, F. Lomeña, F. Lozano, M. Martín, A. Martínez, J. Mas, J.A.M. Mezquita, R. Oliva, A. Prats, E. Pérez, M.J. Rodríguez, A. Trilla y R. Valero.

Recibido: 19.11.18

Aceptado: 26.11.18

Conflicto de intereses: No declarado.

Competing interests: None declared.

(C) 2019 FEM 


\section{Introducción}

El proceso de adaptación al Espacio Europeo de Educación Superior ha comportado en las universidades españolas y en las facultades de medicina, al menos en teoría, la implantación de la enseñanza basada en competencias y, con ello, la necesidad de proceder a su evaluación [1]. Así mismo, se ha introducido el concepto de evaluación continuada como sistema para acreditar la adquisición de las diferentes competencias [2].

El concepto de evaluación continuada se enmarca en el paradigma de la evaluación para el aprendizaje [3,4], en contraposición con el tradicional concepto de la evaluación del aprendizaje. El concepto de la evaluación para el aprendizaje se inserta de pleno en el proceso educativo, pone el énfasis en que la evaluación tiene valor en tanto en cuanto facilita al estudiante feedback sobre su rendimiento y progreso a lo largo del proceso formativo, y le permite analizar sus errores y tomar medidas para su corrección. Presenta la ventaja de que facilita una gran cantidad de información durante el proceso educativo y sirve para dirigir y estimular el aprendizaje de cada estudiante en particular. La evaluación continuada implica que cada actividad docente vaya seguida de una actividad de evaluación, utilizando diferentes instrumentos evaluativos y de la correspondiente retroalimentación al estudiante.

La competencia no es genérica, sino especifica. Un estudiante puede rendir bien en un examen concreto, en un momento determinado, pero nadie asegura que lo haga igual de bien en otro momento. Por ello, una sola medida - una sola prueba- no es suficiente y toda evaluación de competencias debe combinar información procedente de diferentes instrumentos y en distintos tiempos [5].

Cualquier decisión que el profesorado deba tomar sobre el rendimiento de un alumno no puede basarse en el resultado de un único momento de evaluación, que por lo general se hace al final del curso, sino que debe basarse en la información exhaustiva que un profesor recibe de los diferentes momentos de evaluación a lo largo de todo el proceso formativo [4]. Por otra parte, cuanto más importante sea la decisión a tomar (p. ej., decidir si un estudiante supera o no una disciplina), no podemos basarnos en los resultados de una única evaluación final, sino en el sumatorio de todos los resultados de las actividades de evaluación y estas actividades deben ser lo más numerosas posibles [6,7]. Solamente un número suficiente de puntos de referencia, estructurados y organizados dentro del programa de la institución, permitirán tomar las decisio- nes coherentes y justas que la sociedad demanda. Por ello, una evaluación continuada realmente eficaz no puede basarse en unas pocas pruebas de tipo eliminatorio como suele ser habitual en nuestro entorno [7].

En los últimos tiempos, expertos en evaluación en educación médica han introducido un nuevo concepto muy relacionado con lo anteriormente descrito: el programa de evaluación [6,7]. La adopción de un programa institucional de evaluación frente a un sistema de evaluación compartimentado se inserta de lleno en el proceso educativo, provee una gran cantidad de información a lo largo de dicho proceso, facilita el aprendizaje (evaluación para el aprendizaje), permite obtener una visión completa global de la competencia del estudiante a partir de la selección cuidadosa de diferentes instrumentos de evaluación, evalúa repetidamente cada dominio competencial en diferentes momentos, aumenta la solidez de las decisiones importantes y provee información para mejorar la enseñanza y el currículo. Sin embargo, por lo general, las facultades de medicina han elaborado planes de estudios sin incluir programas integrales de evaluación. Se ha dejado la evaluación en manos de los responsables de cada asignatura, con lo cual solo se dispone en general de evaluaciones compartimentadas y no se lleva a cabo una adecuada evaluación de competencias.

Actualmente, el Grado de Medicina en la Facultad de Medicina y Ciencias de la Salud de la Universitat de Barcelona consta de 360 créditos ECTS distribuidos en seis cursos anuales. Los tres primeros cursos comprenden asignaturas predominantemente de formación básica y no integradas (Fig. 1), mientras que los tres últimos cursos se componen de forma casi exclusiva de asignaturas de formación clínica con cierto grado de integración. El grado se imparte en dos campus (Clínic y Bellvitge). En el caso del Campus Clínic, cada curso consta de dos grupos de aproximadamente 90 estudiantes cada uno. La adaptación al proceso de Bolonia se ha llevado a cabo en las diferentes asignaturas de forma relativamente independiente y con poco intercambio de experiencias entre ellas. La implementación del nuevo plan de estudios ha supuesto también cambios en los procesos de evaluación y la introducción, al menos en teoría, de la evaluación continuada.

El objetivo de este trabajo ha sido describir y analizar, una vez se han cumplido seis años de la implementación del nuevo plan de estudios, cómo se está llevando a cabo el proceso de evaluación continuada en las asignaturas de los tres primeros cursos del Grado de Medicina en el Campus Clínic, qué instrumentos se están utilizando para ello, y conocer la 
satisfacción de los coordinadores de cada asignatura con las estrategias de evaluación que están empleando. Se comparan las prácticas evaluativas con las recomendadas por la investigación en evaluación en educación médica y, a partir de dicha comparación, se plantean propuestas de mejora.

\section{Sujetos y métodos}

Se concertó una cita con los coordinadores de todas las asignaturas troncales $(n=23)$ de los cursos primero, segundo y tercero de dicho grado, a los cuales se solicitó cumplimentar presencialmente una encuesta que constaba de un apartado de datos objetivos sobre las actividades de evaluación y una parte anónima de valoración y opinión de estas. Las encuestas se realizaron durante el otoño de 2016 y se refirieron a las asignaturas del curso 2015-2016. El $100 \%$ de los coordinadores contestaron la encuesta.

Los datos de tipo cuantitativo (porcentaje de nota, valoración en una escala de 1 a 5) se analizaron mediante estadística descriptiva. Las respuestas abiertas de valoración de las asignaturas se anonimizaron y se desglosaron en ítems semánticos simplificados, que se representaron gráficamente mediante el programa Word Cloud Generator.

\section{Resultados}

\section{Actividades de evaluación acreditativa. Características y ponderación en la nota final}

Las actividades de evaluación acreditativa se agruparon en cinco grandes clases: prueba de síntesis, pruebas parciales, pruebas de evaluación de prácticas o seminarios, trabajos autónomos y otras actividades. La figura 1 resume las actividades utilizadas en cada una de las asignaturas. La tabla y la figura 2 muestran la ponderación de las diferentes actividades en la nota final de las asignaturas.

\section{Prueba de síntesis}

Prácticamente todas las asignaturas (22 de 23) realizan una prueba de síntesis que es obligatoria en 21 de ellas. En la gran mayoría de asignaturas (20 de 23), el peso de esta prueba se encuentra en el rango del $50-65 \%$ de la nota final (Figs. 1 y 2), suponiendo de media un $57 \%$ de la nota final.

Se trata en todos los casos de una prueba individual y escrita que evalúa los contenidos tratados en todas las actividades de la asignatura. Aunque en la encuesta no se preguntaba por el tipo de preguntas
Figura 1. Resumen de las actividades de evaluación acreditativa utilizadas en las asignaturas troncales de los tres primeros cursos del Grado de Medicina de la Universitat de Barcelona (Campus Clínic). En algunos casos se indica entre paréntesis el número de pruebas de cada tipo que se realizan en una asignatura. En algunas asignaturas se evalúan actividades que pueden subir la nota, de tal manera que la nota final puede ser teóricamente superior a 10

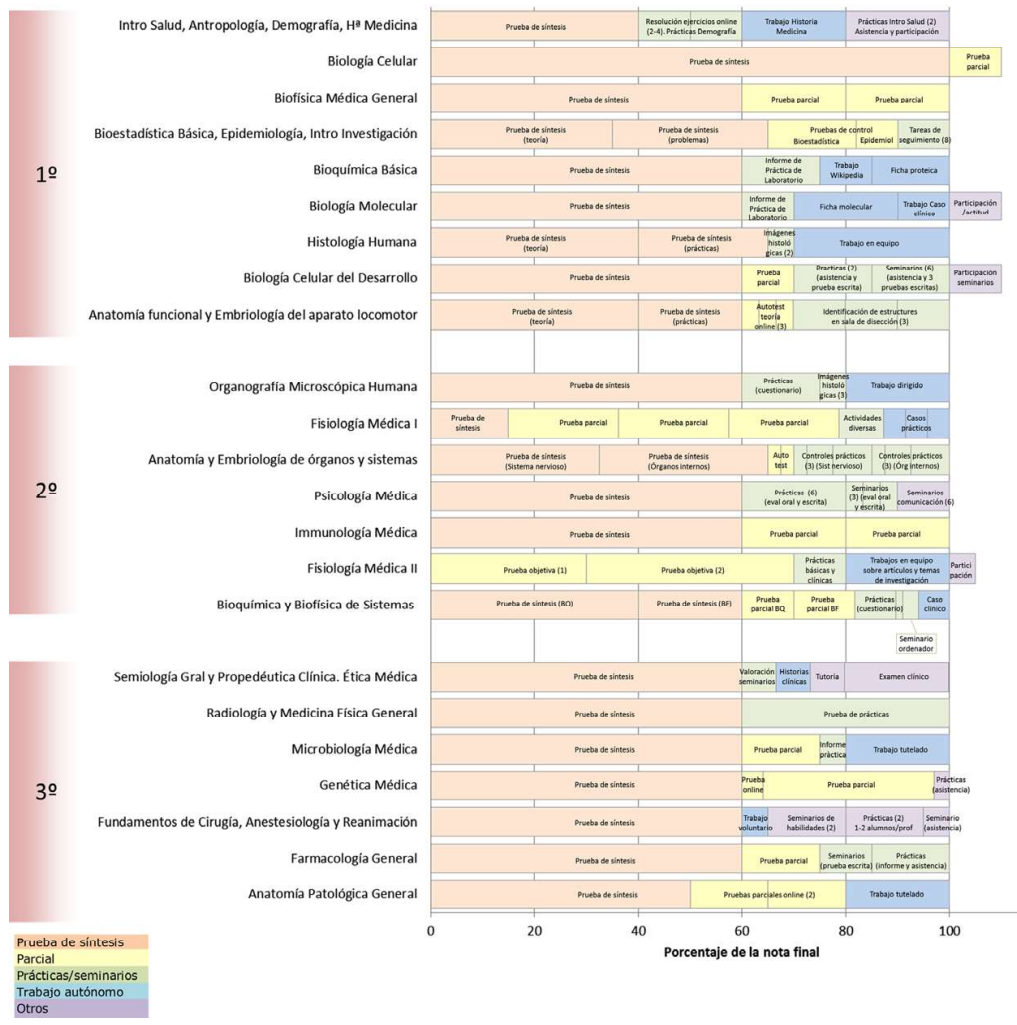

planteadas en estas pruebas, muchos coordinadores quisieron poner de manifiesto las estrategias que habían adoptado para intentar mejorar la efectividad de estas pruebas, como preguntas abiertas (no test), preguntas tipo test con más de una opción correcta, preguntas de integración de conceptos o preguntas que requieren madurez y reflexión. En la mayoría de asignaturas, las pruebas de síntesis contienen preguntas de tipo test con cuatro o cinco opciones y una respuesta correcta, si bien muchas incluyen también preguntas de tipo abierto. En varias asignaturas, la prueba de síntesis consta de dos partes claramente diferenciadas (teoría y prácticas-problemas), de un valor similar respecto a la nota final.

\section{Pruebas parciales}

Las pruebas parciales son pruebas escritas e individuales donde se evalúan, típicamente a mitad de 
Figura 2. Peso de los diferentes tipos de actividades en la nota final en los tres primeros cursos del grado.

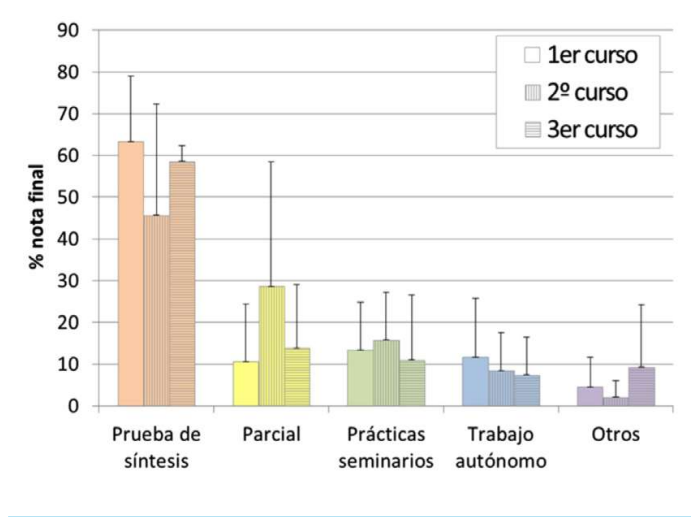

curso, los contenidos de una parte de la asignatura. Se realizan en 14 asignaturas y suponen como media un $17 \%$ de la nota final, si bien encontramos asignaturas donde estas pruebas suponen más del $40 \%$ de la nota final $(n=4)$ y otras donde suponen menos del 15\% $(n=6)$. El número de pruebas parciales en estas asignaturas es de uno $(n=4)$, dos $(n=8)$ o tres $(n=2)$.

En cuatro casos, los coordinadores manifestaron que estas pruebas no liberan materia (en los demás casos no se dispone de este dato). Finalmente, cuatro asignaturas realizan pruebas parciales online que tienen repercusión en la nota final. Algunas asignaturas hacen pruebas online como evaluación formativa que no tienen peso en la nota final, pero dado que no se preguntó específicamente, no se dispone del dato de cuántas asignaturas hacen estas pruebas.

\section{Pruebas de evaluación de prácticas o seminarios} La mayoría de las asignaturas, si no todas, incluyen en la prueba de síntesis preguntas sobre prácticas y seminarios. Algunas incluyen en la prueba de síntesis un apartado claramente diferenciado de preguntas sobre prácticas, seminarios o problemas (Fig. 1). Además, muchas asignaturas $(n=17)$ realizan pruebas escritas $\mathrm{u}$ orales específicas para evaluar estas actividades. Estas pruebas suponen de media un $13 \%$ de la nota final, siendo este porcentaje superior al $30 \%$ en cinco asignaturas.

Estas pruebas son muy heterogéneas en varios aspectos. Lo son en cuanto al tipo de prueba: de identificación de estructuras anatómicas o histológicas, informes de prácticas, cuestionarios tipo test o con preguntas abiertas, entre otras. Son también heterogéneas en cuanto al número de pruebas de
Tabla. Porcentaje de la nota final que suponen los diferentes tipos de evaluación acreditativa (media \pm desviación estándar) y número de asignaturas que utilizan los diferentes tipos de actividades.

\begin{tabular}{lcc}
\hline & $\%$ nota final & Asignaturas \\
\hline Prueba de síntesis & $56,5 \pm 18,5$ & 22 \\
\hline Pruebas parciales & $17,1 \pm 20,9$ & 14 \\
\hline Evaluaciones de prácticas/seminarios & $13,4 \pm 12,4$ & 17 \\
\hline Trabajos autónomos & $9,4 \pm 11,0$ & 12 \\
\hline Otras actividades de evaluación & $5,2 \pm 9,6$ & 7 \\
\hline
\end{tabular}

este tipo que hacen las diversas asignaturas: hallamos cinco asignaturas con una o dos pruebas de este tipo y otras cinco con siete o más pruebas.

\section{Trabajos autónomos}

Se trata de trabajos más o menos dirigidos por los profesores en los que se tratan en profundidad algunos aspectos específicos, muy a menudo aspectos clínicos, relacionados con la asignatura.

Cerca de la mitad de las asignaturas $(n=12)$ tienen algún trabajo de este tipo, generalmente una actividad por asignatura $(n=9)$. Suponen de media un $9 \%$ de la nota final, si bien en ocho asignaturas este porcentaje es superior al $20 \%$.

Los trabajos autónomos son el contexto en el cual más a menudo se evalúan las competencias del trabajo en equipo y de la expresión oral. Así, en ocho asignaturas, estos trabajos se hacen en equipo, mientras que en seis la presentación del trabajo se hace oralmente (cuatro, sólo oral, y dos, oral y por escrito).

\section{Otras actividades de evaluación acreditativa}

En este apartado se han incluido actividades de evaluación acreditativa que no encajan en ninguno de los otros cuatro grupos. Es, por tanto, un grupo bastante heterogéneo. Hemos identificado actividades de este tipo en ocho asignaturas que suponen de media un $5 \%$ de la nota final, pero mientras que en tres asignaturas estas actividades suponen más de un $20 \%$ de la nota final, en las otras cinco suponen menos de un $15 \%$.

En este apartado se incluyen, por un lado, actividades donde se evalúan de forma más individualizada habilidades profesionales como, por ejemplo, el examen clínico con paciente real en la asignatura de semiología, las prácticas de cirugía y anestesiología con 1-2 alumnos por profesor en la asignatura 
de fundamentos clínicos, los seminarios de comunicación en psicología médica o las prácticas en introducción a la salud. Son actividades que se encuentran en las asignaturas más clínicas de los tres primeros cursos y que predominan en las asignaturas de los cursos posteriores.

Por otra parte, algunas asignaturas valoran aspectos como la asistencia a determinadas actividades, la participación o la actitud. En algunos casos, estas valoraciones suponen una calificación extra con el objetivo de premiar a alumnos especialmente participativos o brillantes.

\section{Valoración de los coordinadores de asignatura sobre las actividades de evaluación acreditativa}

Valoración de las actividades en una escala de 1 a 5 En cuanto a la valoración por parte de los coordinadores de las diferentes actividades en una escala de 1 (muy poco satisfecho) a 5 (muy satisfecho), todas las actividades se valoraron con puntuaciones de 3 , 4 o 5 . El tipo de actividades mejor valoradas en conjunto fueron los trabajos autónomos $(4,3 \pm 0,8 ; n=$ $12)$, seguidos de las actividades englobadas en el bloque de 'otras actividades' $(4,1 \pm 0,5 ; n=6)$ y de las pruebas de síntesis $(4,0 \pm 0,6 ; n=22)$. Las actividades menos valoradas en conjunto fueron las pruebas parciales $(3,7 \pm 0,7 ; n=14)$ y las pruebas de evaluación de prácticas o seminarios $(3,5 \pm 0,4 ; n=17)$.

\section{Respuestas abiertas: puntos débiles/puntos a mejorar} A partir de las respuestas abiertas a la pregunta 'puntos débiles/puntos a mejorar', identificamos 82 ítems/frases cortas, resumidos visualmente en la figura 3. Doce de ellos corresponden a lo que podríamos llamar 'deseos'. Se menciona en este sentido que 'sería deseable hacer más actividades de evaluación, 'más pruebas orales', 'más prácticas', más seminarios,' 'más pruebas de autoevaluación', 'integrar mejor teoría y actividades', 'hacer pruebas de síntesis con preguntas cortas' o 'con preguntas orales' o 'más integradoras de los conocimientos. La razón aducida más habitualmente para no llevar a cabo estos 'deseos' en la actualidad es la falta de tiempo y el elevado número de estudiantes.

El punto débil repetidamente más mencionado por los coordinadores fue el número demasiado elevado de alumnos, y ligado con ello, el tiempo que se necesita para llevar a cabo actividades más elaboradas o en grupo pequeño cuando se trabaja con grupos tan numerosos (90 alumnos $\times 2$ clases).

En cuanto a los puntos débiles de las actividades de evaluación que están utilizando, los coordinadores mencionaron:
Figura 3. Representación gráfica de las respuestas abiertas aportadas por los coordinadores a la pregunta 'puntos débiles/puntos a mejorar del sistema de evaluación utilizado en la asignatura que coordina'.

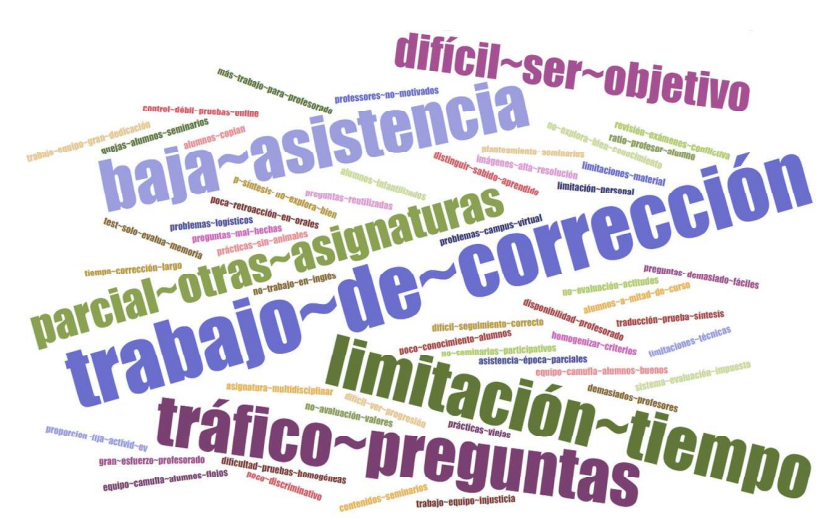

- El hecho de no evaluar actitudes o valores, de no utilizar el inglés o de no hacer más seminarios participativos.

- El hecho de que los alumnos copien, el tráfico de preguntas y el débil control en las pruebas escritas fuera del aula.

- Se mencionaron debilidades de actividades de evaluación concretas:

a) Algunas pruebas se consideraba que no discriminan lo suficiente' (se atribuye en parte al tráfico de preguntas).

b) 'La dificultad de tener criterios homogéneos en pruebas donde participan muchos profesores'

c) 'La corrección de muchas pruebas hace difícil ser objetivo'.

d) 'Las pruebas en equipo pueden dar lugar a injusticias, camuflar tanto a los alumnos brillantes como a los flojos'.

e) En un caso se manifestó que 'la prueba de síntesis no explora de la mejor manera el conocimiento'

f) En algunos casos se autocriticó la utilización de preguntas viejas, reutilizadas, mal hechas, demasiado fáciles o que no discriminan la memorización de la comprensión.

g) Se manifestó la dificultad de distinguir lo que ya sabían previamente los alumnos de lo que habían aprendido durante el curso.

h) Se autocriticó que en las pruebas orales se da al alumno poca justificación de la nota, poca retroacción.

i) Se manifestaron quejas por el trabajo que supone la traducción de pruebas de síntesis largas o porque algunas revisiones de pruebas de síntesis son conflictivas. 
Aunque se pedía a los coordinadores que enunciaran puntos débiles o aspectos a mejorar del sistema de evaluación utilizado en su asignatura, muchos de ellos quisieron manifestar puntos débiles o a mejorar no tanto del sistema de evaluación como de la asignatura, de los alumnos o del sistema en general Como debilidades en este apartado se mencionaron:

- Alumnos: 'baja asistencia' y 'alumnos infantilizados'.

- Profesores: 'demasiados profesores por asignatura,' 'poco motivados', 'poco disponibles'.

- Asignaturas: 'demasiado multidisciplinar,' 'demasiados profesores', 'no está en el curso correcto'.

- Otras asignaturas: en algún caso se mencionó que 'pruebas de evaluación de otras asignaturas en mitad de curso generan conflictos, principalmente de asistencia.

- Técnicos: se mencionaron problemas técnicos o logísticos (sin concretar), 'problemas técnicos en el campus virtual' (aplicación Moodle de la universidad), 'problemas con imágenes de alta resolución en las pruebas de evaluación.

- Generales: 'poco conocimiento de los alumnos por parte de los profesores', 'incapacidad de hacer un seguimiento correcto' (atribuido al número de alumnos), 'el hecho de tener porcentajes fijos de dedicación a algunos tipos de actividades', 'el hecho de que esto sea impuesto', 'el no «creer» en la actividad continuada'.

- Varios: quejas por 'los alumnos que empiezan a medio curso'.

\section{Respuestas abiertas: puntos fuertes/puntos a destacar}

De las respuestas abiertas a la pregunta sobre 'puntos fuertes/puntos a destacar' identificamos 100 ítems/ frases cortas que se muestran de forma gráfica en la figura 4.

Varios coordinadores mencionaron como puntos fuertes aspectos relacionados con los alumnos: 'los alumnos aprenden', 'están contentos', 'están motivados,' 'valoran bien la asignatura, 'son conscientes de su progreso,' 'el sistema de evaluación es respetuoso con el estudiante'

También se mencionaron como puntos fuertes los resultados obtenidos: buenos resultados, nota media muy alta, pocos suspensos, ningún suspenso. En este sentido es interesante como contrapunto el comentario de un coordinador que manifestaba que 'prácticamente el 100\% de los alumnos aprueba la asignatura y esto ayuda a que los alumnos la valoren bien, pero en mi opinión, aprobar es demasiado fácil'.

Entrando ya en aspectos valorados como puntos fuertes/puntos a destacar del sistema de evaluación, los coordinadores consideraron como positivo que el sistema utilizado es compensado/bien ponderado, variado, objetivo, justo, transparente, coherente, exigente, completo/evalúa muchos ítems, da una visión de conjunto, refleja bien los conocimientos adquiridos, discrimina, no evalúa sólo memoria, evalúa competencias puras, es práctico, funciona, requiere un trabajo constante por el estudiante y cumple la normativa de la universidad.

Muchos coordinadores valoraron positivamente aspectos relacionados con la prueba de síntesis. A menudo se mencionó el hecho de que la preparación de la prueba de síntesis requiere mucho esfuerzo y, en este sentido, nuestra apreciación personal fue que los coordinadores realizan en general un trabajo constante de adaptación y mejora de estas pruebas. Se citó en concreto el hecho de utilizar preguntas no test que discriminan mejor que las de tipo test o preguntas que evalúan mucho más que conocimiento memorístico. Igualmente, se valoraron determinadas actividades en relación a la prueba de síntesis, como el hecho de que la prueba parcial familiariza al estudiante con la posterior prueba de síntesis o que las actividades de evaluación continuada hacen subir la nota final.

Finalmente, numerosos coordinadores valoraron positivamente aspectos de actividades de evaluación concretas como:

- El hecho de que determinadas actividades evaluadas suponen una aproximación al trabajo experimental, a la investigación y al laboratorio.

- El hecho de que determinadas actividades evaluadas fomentan la discusión, la participación, la reflexión, la selección y análisis de información científica y el trabajo con fuentes de información reales y de uso profesional.

- El hecho de que los estudiantes creen documentos originales, dibujen, trabajen en equipo o hagan presentaciones orales.

- Aspectos de tipo práctico como la corrección instantánea de algunas pruebas, el hecho de que sean fáciles de preparar por el profesorado, de que se minimicen las 'fugas' de preguntas o las repeticiones de preguntas de un año a otro.

- El hecho de que se trabaje en equipos docentes y de que se preparen preguntas consensuadas.

\section{Discusión}

Este estudio de tipo descriptivo ha pretendido analizar cómo se está realizando actualmente la evaluación acreditativa de los aprendizajes en las asignaturas de los tres primeros cursos del Grado de Me- 
dicina en uno de los campus de la Facultad de Medicina y Ciencias de la Salud de la Universitat de Barcelona. De los resultados obtenidos se deduce que, en la mayoría de las asignaturas obligatorias de los tres primeros cursos de dicho grado, existe una gran diversificación en las actividades de evaluación acreditativa, aunque podríamos establecer que una asignatura tipo realizaría una prueba de síntesis al final del período formativo que supondría un $60 \%$ de la nota final, una o dos pruebas parciales, una o dos actividades de evaluación de seminarios o prácticas y un trabajo autónomo.

$\mathrm{Al}$ analizar este sistema de evaluación tipo y compararlo con lo que entienden por evaluación continuada los expertos en evaluación en educación médica $[2,3]$, podemos convenir que si bien los planes docentes de las asignaturas establecen que se realiza una evaluación continuada, el tipo de evaluación que se realiza no puede considerarse en la mayoría de los casos una evaluación de este tipo. La evaluación continuada supone efectuar diversas actividades evaluativas a lo largo del programa formativo que permiten dar feedback al estudiante y le ayuden a adoptar medidas para la mejora de su aprendizaje. Una evaluación como la que se realiza en esta facultad, que de media supone la realización de una o dos pruebas parciales de evaluación y una prueba final de síntesis con una ponderación media de un $60 \%$, dificulta apreciar en cada momento el progreso del estudiante y no provee oportunidades de mejora al estudiante. En este aspecto, sería más adecuado no someter a los alumnos a múltiples actividades evaluativas durante el curso si al final la evaluación final o de síntesis sigue teniendo una ponderación excesiva y superior al resto de las actividades, ya que no dejaríamos de estar de nuevo ante un examen final.

El estudio evidencia la existencia de una gran variabilidad en las actividades evaluativas que llevan a cabo las diferentes asignaturas, pero estas actividades no son conocidas por los profesores de las distintas asignaturas, con lo cual, al final, cada una de ellas puede llegar a evaluar aquellos conocimientos, habilidades y actitudes específicos que les son propios para la adquisición de determinadas competencias, pero no son conocedores de cómo se evalúan los conocimientos, habilidades y actitudes propios de otras materias que contribuyen también a la adquisición de la misma competencia. Es decir, nos encontramos ante un tipo de evaluación muy compartimentada que dificulta enormemente la evaluación de las competencias, situación que los expertos aconsejan evitar [8].

Una competencia no es un patrimonio exclusivo de una sola asignatura, sino que por lo general con-
Figura 4. Representación gráfica de las respuestas abiertas aportadas por los coordinadores a la pregunta 'puntos fuertes/puntos a destacar del sistema de evaluación utilizado en la asignatura que coordina'.

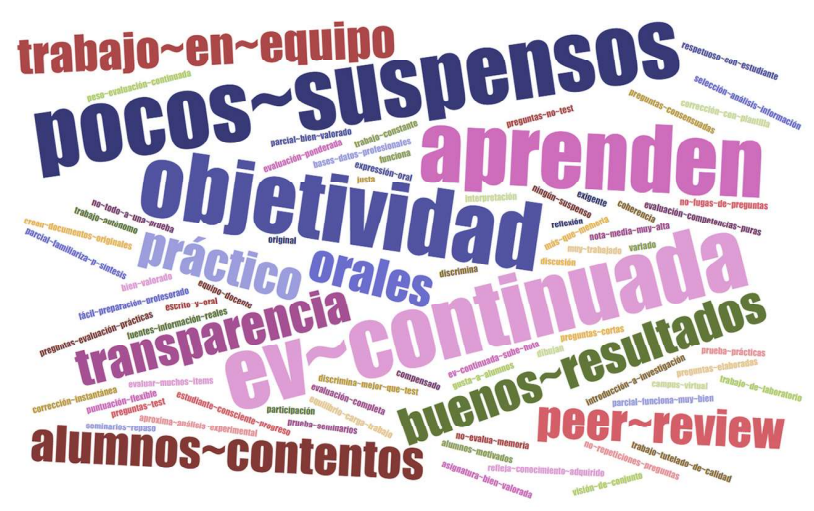

tribuyen a ella distintas materias o asignaturas [1]. Por tanto, el sistema compartimentado de evaluación nos aleja del concepto de evaluación programática o de programa de evaluación institucional y longitudinal que propugna la investigación educativa en este campo y que permitiría llevar a cabo una adecuada evaluación de competencias [4,6]. Por ello, sería recomendable que las facultades de medicina se encaminaran progresivamente hacia un modelo de evaluación institucional que obviase estas dificultades. Sin embargo, no se puede negar que establecer una evaluación de este tipo comporta dificultades y resistencias.

El trabajo pone de manifiesto que varias asignaturas realizan actividades para evaluar competencias transversales, como los trabajos en equipo, las presentaciones orales y las evaluaciones por iguales. Es muy meritorio que se realicen este tipo de actividades, dada la gran carga de trabajo que comportan. Sin embargo, y puesto que son competencias transversales, sería recomendable que las diferentes materias establecieran acuerdos con el fin de trabajar y evaluar estas competencias transversales con actividades evaluativas conjuntas, y evitar así sobrecargar al estudiante con múltiples actividades que pretenden los mismos objetivos.

Los resultados también evidencian, en general, una fuerte implicación de los coordinadores en su tarea y la realización de una constante revisión de las actividades de evaluación. Sin embargo, parecen existir grandes diferencias en el grado de convicción por parte del profesorado sobre las virtudes de la evaluación continuada o la evaluación de competencias. Pensamos que este hecho podría atribuirse 
al desconocimiento, por un importante número de profesores, del concepto de lo que es una verdadera evaluación continuada y de lo que supone una verdadera evaluación de competencias. De ahí surge la necesidad de que los profesores se formen en los procesos de evaluación, en los principios fundamentales de la evaluación en educación médica y de los distintos instrumentos y sus características. La necesidad de establecer programas formativos para el profesorado sobre esta temática es una de las recomendaciones propuestas desde siempre por los expertos en educación médica [8].

Establecer un sistema real de evaluación continuada comporta dificultades. De entrada, el hecho de que las diferentes asignaturas se impartan en un formato semestral, que en muchos casos se reduce a un período lectivo efectivo de cuatro meses, disminuye las posibilidades de realizar una adecuada evaluación continuada. Por otra parte, la dedicación del profesorado es muy desigual porque habitualmente es poco relevante el reconocimiento institucional a los profesores que utilizan estrategias innovadoras y que suponen, por tanto, un mayor esfuerzo.

Una limitación importante del trabajo es no haber realizado un estudio acerca de las percepciones de los alumnos sobre el sistema de evaluación. Sus opiniones serían muy útiles para analizar el problema, sobre todo teniendo en cuenta que el alumno es un elemento clave en todo sistema evaluativo.

En conclusión, a pesar del importante esfuerzo realizado por las diferentes asignaturas y sus coordina- dores para mejorar las actividades de evaluación, no estamos en la situación más adecuada. Los resultados del análisis deberían llevar a reflexionar sobre las posibilidades de realizar cambios en el sistema de evaluación, valorar la conveniencia de que todas las asignaturas tuvieran un mismo patrón de evaluación y, sobre todo, si sería adecuado y posible establecer un programa institucional de evaluación que implicase a todas las asignaturas, abandonando la actual evaluación compartimentada. Así mismo, debería llevar a repensar conjuntamente lo que ha de suponer la evaluación continuada real y el papel que ha de tener en ella la prueba de síntesis.

\section{Bibliografía}

1. Palés-Argullós J, Nolla-Domenjó M, Oriol-Bosch A, Gual A. Proceso de Bolonia (I). Educación orientada a competencias. Educ Med 2010; 13: 127-35.

2. Gual A, Palés-Argullós J. La evaluación continua: una rara avis. Educ Med 2012; 15: 185-8.

3. Martinez ME, Lipson JI. Assessment for learning. Educ Leader 1989; 47: 73-5.

4. Schuwirth LW, Van der Vleuten CP. Programmatic assessment: from assessment of learning to assessment for learning. Med Teach 2011; 33: 478-85.

5. Van der Vleuten CP, Schuwirth LW. Assessment of professional competence: from methods to programmes. Med Educ 2005; 39: 309-17.

6. Van der Vleuten CP, Schuwirth LW, Driessen EW, Dijkstra J, Tigelaar D, Baartman LK, et al. A model for programmatic assessment fit for purpose. Med Teach 2012; 34: 205-14.

7. Van der Vleuten CP, Schuwirth LW, Driessen EW, Govaerts MJB, Heeneman S. Twelve tips for programmatic assessment. Med Teach 2015; 37: 641-6.

8. Harden RM. Ten key features of the future medical school -not an impossible dream. Med Teach 2018; Oct 16. [Epub ahead of print]. 